$\underset{Y 12}{B W X T}$

A BWXT/Bechtel Enterprise

\section{$\mathrm{Y}-12$ \\ NATIONAL SECURITY COMPLEX}

\title{
Brighter Screens for Nondestructive Digital X-ray Radiography
}

\author{
A.C. Miller, Z.W. Bell, D.A. Carpenter \\ Technology Development Division \\ Y-12 National Security Complex
}

September 15, 2003

\author{
Prepared by the \\ Y-12 National Security Complex \\ Oak Ridge, Tennessee 37831-8169 \\ managed by \\ BWXT Y-12 \\ for the \\ U. S. DEPARTMENT OF ENERGY \\ under contract DE-ACO5-00OR22800
}




\section{DISCLAIMER}

This report was prepared as an account of work sponsored by an agency of the United States Government. Neither the United States Government nor any agency thereof, nor any of their employees, makes any warranty, express or implied, or assumes any legal liability or responsibility for the accuracy, completeness, or usefulness of any information, apparatus, product, or process disclosed, or represents that its use would not infringe privately owned rights. Reference herein to any specific commercial product, process, or service by trade name, trademark, manufacturer, or otherwise, does not necessarily constitute or imply its endorsement, recommendation, or favoring by the United States Government or any agency thereof. The views and opinions of authors expressed herein do not necessarily state or reflect those of the United States Government or any agency thereof. 
Y/DW- 1922

\title{
Brighter Screens for Nondestructive Digital X-ray Radiography
}

\author{
A.C. Miller, Z.W. Bell, D.A. Carpenter \\ Technology Development Division \\ Y-12 National Security Complex
}

September 19, 2003

Prepared by the

Y-12 National Security Complex

Oak Ridge, Tennessee 37831-8169

managed by BWXT Y-12

for the

U. S. DEPARTMENT OF ENERGY

under contract DE-ACO5-00OR22800 


\title{
Brighter Screens for Nondestructive Digital X-ray Radiography
}

\author{
A.C. Miller, Jr. \\ Z.W. Bell \\ D.A. Carpenter \\ Technology Development Division
}

\section{SUMMARY}

Fine resolution, bright $\mathrm{X}$-ray screens are needed for digital radiography and material characterization at the Y-12 National Security Complex (Y-12). Current technology is simply not adequate for transferring high-energy $\mathrm{X}$-ray images to visible light for demanding digital applications. Low energy radiography and especially emerging tomographic technologies are severely hampered for Y-12 nondestructive evaluation (NDE) applications by dim screens with poor resolution. Also, the development of more advanced materials characterization techniques, such as electron backscatter diffraction (EBSD), is driven by a design agency desire for tighter specifications and more uniform materials. Brighter screens would allow us to probe materials on a finer scale, leading to a better understanding of material behavior.

A number of X-ray screen materials were studied that would be suitable for direct replacement in existing digital imaging systems. Spectroscopic evaluations were first made for a several candidates and indicated that lutetium orthosilicate (LSO) would be a promising candidate for $\mathrm{MeV}$ images. A relative comparison of brightness at various energies was then completed which showed that cesium iodide (CsI) could increase brightness by over an order of magnitude. Since image quality is also important for better screens, the resolving capabilities of candidate materials were measured. Resolution measurements were completed at X-ray peak energies up to $420 \mathrm{KeV}$ with magnified optical imaging systems, and indicated that LSO and Industrial Quality Incorporated glass (IQI) exhibited higher resolution than the CsI screen. The results give a choice of materials that can be tailored to the particular test under consideration. If high-speed images are necessary and some resolution can be sacrificed, the CsI screen will be a good choice. The screen can be replaced by an IQI or LSO unit if higher resolution is needed later, for instance to focus in on a region of interest.

\section{INTRODUCTION}

Y-12 Product Certification organization, in support of many plant operations, is actively pursuing a radiography improvement program that includes extensive use of digital technologies. Researchers within the organization have expressed a keen interest in brighter, higher quality screens to make promising new digital methods possible for several challenging Y-12 applications. The current radiography practice at Y-12, however, is still film-based. These methods provide high-resolution images, but are labor-intensive, time-consuming, difficult to share with several viewers, and generate 
significant amounts of hazardous wastes. Also, X-ray scatter mechanisms can severely degrade film images at high energies. Digital methods can overcome many of these deficiencies, but presently lack the high resolution.

Only a few materials are now commercially available for scintillators in the energy ranges of interest. One of the best products is a glass produced by Industrial Quality, Inc. (IQI) which uses small amounts of Terbium in its mix to produce green light. This glass was not formulated for high-energy X-ray use and therefore produces poor quality images even with much effort. Efficient scintillating screens are needed over a wide range of energies and are a key component to effective digital radiography systems for Y-12 applications.

Major improvements in charge coupled device (CCD) imaging technology have made remarkable changes in scientific fields such as spectroscopy and astronomy. Also, with the revolution in digital computing, processing of massive NDE files and tomographic reconstructions are more easily accomplished. The critical missing link in successfully applying these new technologies to Y-12 applications is an efficient imaging screen. Therefore, to realize intuitive, 3-D imaging of components and assemblies, digital pictures must be acquired much faster and contain much more detail.

Advances in scintillator technology have produced plastic and flexible rubber materials whose emission characteristics can be tailored to match the absorption curve of photosensors. The combinations of potential scintillating materials number in the hundreds. All will have deficiencies since the screen needs to be optimized for so many factors. Plastic and rubber scintillators emitting blue, blue-green, and green are readily available and can be cast in thin films to minimize the depth-of-field blur caused by thick screens.

Microchannel plate image intensifiers have also improved over past vidicon-based units. These amplifiers convert light (at wavelengths from the ultraviolet through the visible) incident on an entrance window to an electron current, amplify the electron current, and convert the amplified current back to light at an exit window while preserving the incident image. Intensity gains of several orders of magnitude are routine for these devices and the "color" of the exit window image need not be the same as that of the incident image. The device serves simultaneously as an amplifier and a wavelength shifter that can match the color of an image produced by a scintillator to the color to which a CCD camera is most sensitive.

Although this project is expected to advance the state of the art, bright X-ray screens will not eliminate radiographic film use at Y-12. It may, however, result in dramatic reduction of film related costs. Of even more importance, however, is the potential gain resulting from acquiring higher quality data and the ability to process many more test units. Brighter, higher quality screens allow a clearer image of the features of interest and pave the way for such innovations as 3-D tomographic images that can be acquired in a reasonable time frame. Tomographic reconstructions reduce much of the training 
required to understand standard radiographs since slices from a 3-D image are easily interpreted.

The major deliverables of the project were experimental data evaluating the relative improvements in resolution and brightness of new screens against the few existing scintillator materials. Deliverables also included evaluation data on a variety of scintillator conversion screens optimized for beam energy.

\subsection{TECHNICAL APPROACH}

A variety of X-ray imaging screens and optical systems were tested that potentially have higher brightness than currently available technology. These screens and systems are direct replacements for present-day equipment, result in higher quality images acquired in shorter times, and make feasible real-time (or at least near-real-time) digital radiography, tomography, and materials characterization.

A short evaluation of available screen materials was first conducted to optimize the selection for the desired energy range. A few of the most promising candidates were procured and tested. Initially, a spectroscopic setup was used to test for brightness and wavelength coupling. Resolution of the screens was evaluated with a microfocus X-ray source and a special, magnified optical system. An illuminating X-ray beam with a very small focus size is important to prevent the measured resolution from being dominated by source-unsharpness effects.

\subsection{MOTIVATION}

Presently, numerous film shots are required to properly set up a new object for X-ray analysis. Although the film costs are not negligible, the major expense is in personnel time. The separation of film exposing, processing, and analysis areas due to environmental requirements has made this problem much worse. New setups in particular can require several iterations to obtain the proper exposure while maximizing contrast and properly aligning the particular feature for analysis. On difficult setups where a critical feature must be inspected, this iteration process has been known to persist for as much as a week or more. Deployment of efficient digital radiography provides immediate feedback to the radiographer to optimize the setup in minimum time.

Indirect costs also compound the problem. Many entries and exits from material access areas are required to expose and analyze the film. Also, a large quantity of environmentally hazardous waste is generated in the film development, accounting for much additional record keeping and disposal cost. Indeed, much of the high-resolution film in use at Y-12 today is no longer even produced domestically. It is important to have proven alternative methods in place in the event that critical supplies become difficult to obtain or quality from after-market vendors becomes suspect. In the past, medical use of film was a sufficiently large market to insure adequate supplies for other 
testing such as NDE. However, digital methods are quickly replacing film use for medical purposes, and this supply may no longer be available.

\subsection{SCREEN BRIGHTNESS EVALUATION}

Brightness and resolution evaluations were performed on a number of promising candidates for X-ray scintillating screens. In the spectroscopic examination of suitable candidates, the response of a photomultiplier tube was measured as a function of wavelength. Several screens were mounted on the tube and exposed to a series of radioactive sources to determine the light output as a function of deposited energy. The number of scintillation photons per deposited $\mathrm{KeV}$ was determined by correcting the calibration curve for the previously measured quantum efficiency of the photomultiplier.

Several of the samples exhibited too slow a decay constant to be measured with the spectroscopic setup electronics. Samples that were tested included a sodium iodide reference sample (NaI), a lutetium orthasilicate (LSO), and cadmium tungstate. The results indicated that the LSO samples could provide greater brightness at high energies than originally thought.

Also, a series of brightness measurements were made on a relative comparison basis. Illumination values from the samples were compared with the currently used scintillator material, IQI $6 \mathrm{~mm}$, under the same X-ray conditions with a charge coupled detector (CCD) that exhibited high response to a broad range of wavelengths. The comparison is shown in Figure 1. Normalization for thickness, density, and index of refraction was not made, so the chart is a relative indicator rather than absolute.

In spite of the limitations to the testing, some important observations can be made. The cesium iodide (CsI) sample with an aluminum screen proved the brightest, and showed an increase of more than 10x over the standard IQI $6 \mathrm{~mm}$ midpoint. Also very bright were the CdWO4 samples. Several scintillating gel materials were also tested. These proved very dim compared to other samples. 


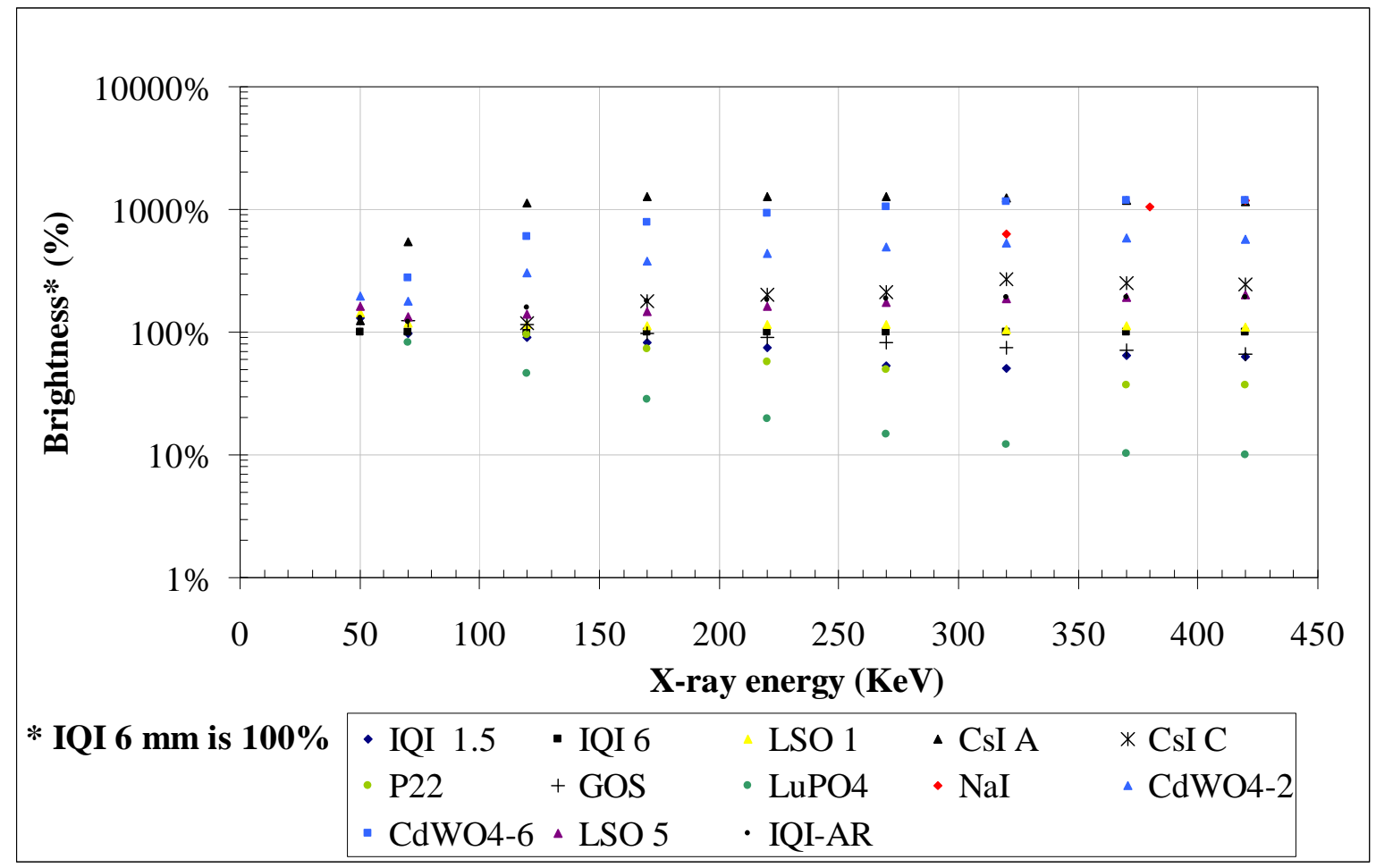

Figure 1. Scintillator brightness evaluation

Most screen materials slowly increased in brightness from 100-400 KeV radiation, and all gave similarly weak responses under $100 \mathrm{KeV}$. The lutetium orthophosphate (LuPO4) sample gave a disappointing response despite customized formulation by material experts at ORNL. The samples' low response at around $50 \mathrm{KeV}$ may be due to the light shield covering the samples and the optical setup. It was determined that the shield begins to attenuate the X-ray beam at lower energies and was a contributor to the low brightness recorded.

Additional testing was performed using a low-energy electron backscatter diffraction imager. Figure 2 shows the qualitative results of 3 different powder screens used to image a silicon crystal under identical conditions. A lutetium orthophosphate (LuPO4), gadolinium oxysulfide (GOS), and phosphor type P22 screens were evaluated. From the images, considerable differences can be discerned in the various screens. A definite increase in brightness is apparent for the P22 phosphor, but at some subjective loss in contrast. Although P22 has a slower response than GOS, special screens made from P22 could be quite useful in the examination of deformed or machined metal surfaces in scanning electron microscopy imaging. Further testing at higher energies, reported in the next section, provided more quantitative analysis of the imaging properties of the various screens. 


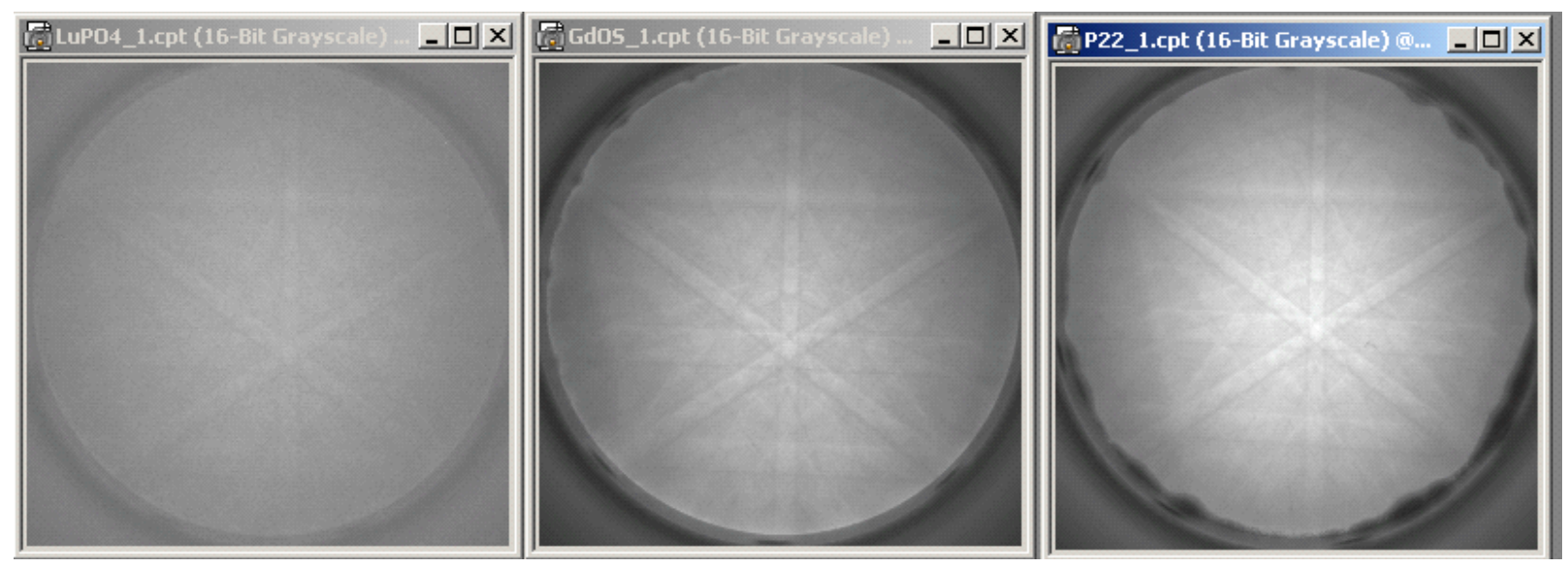

Figure 2. Electron Backscatter Diffraction Screen Images

\subsection{SCREEN RESOLUTION TESTING}

Evaluation of screen resolution proved much more challenging than measuring screen brightness. A series of imaging devices were designed and constructed to provide adequate magnification for the screen evaluation. To achieve high magnification, light gathering power was sacrificed, and some samples proved to dim to obtain accurate data, especially at energies around $50 \mathrm{KeV}$ and lower. Suitable devices were constructed that were capable of viewing a $20 \mathrm{lp} / \mathrm{mm}$ target and used to measure the resolving capabilities of the various materials.

Typical resolution target images are seen in Figures 3-6 using white light images compared to the X-ray illuminated scintillator screen. Figures 3 and 5 were taken in ambient light without the $\mathrm{X}$-ray screens in place. The screen used for the samples was a CsI screen with a Carbon filter (CsI C). Resolution appears to wash out at about 14-16 $\mathrm{lp} / \mathrm{mm}$ for the converging line pair scintillator screen image and is slightly better with the line pair target. Although some image degradation is apparent in the scintillator screen images, the pictures demonstrate that high-resolution digital radiography can be obtained with commercial screens.

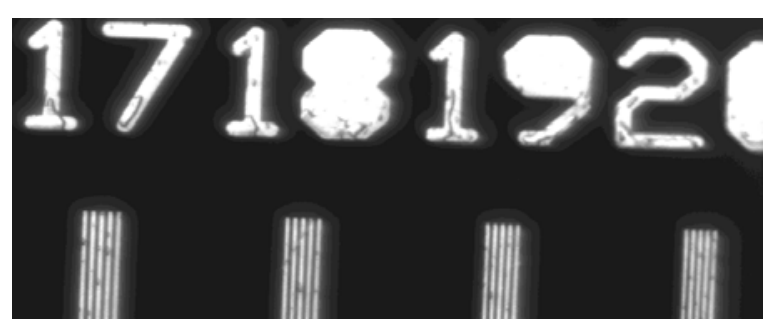

Figure 3. Line Pair Target - White Light

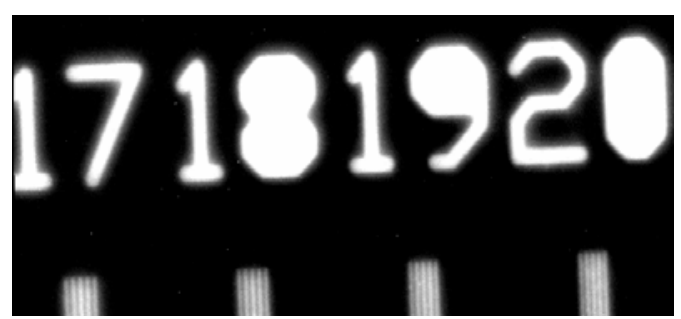

Figure 4. Line pair target - Scintillator Screen 


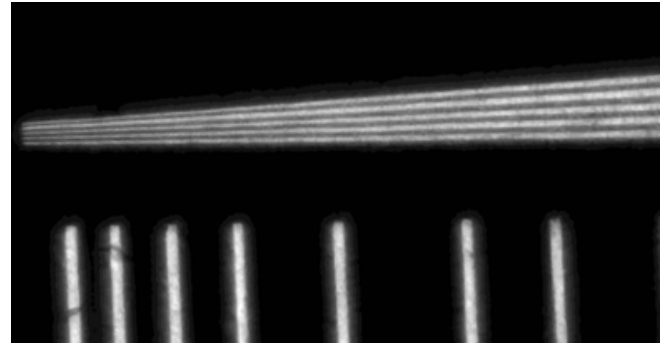

Figure 5. Converging Target - White Light

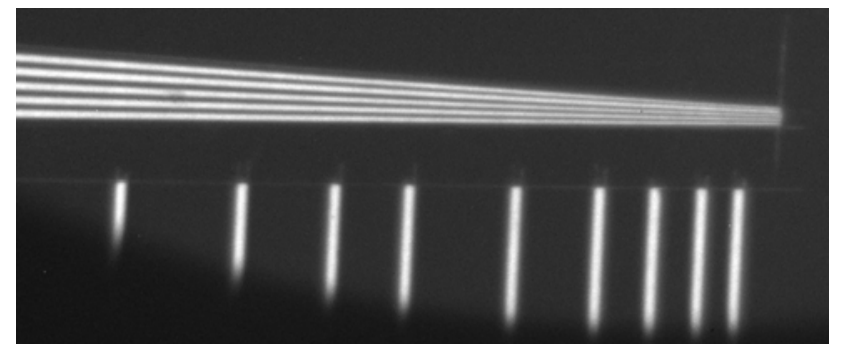

$10 \mathrm{lp} / \mathrm{mm}$

$20 \mathrm{lp} / \mathrm{mm}$

Figure 6. Converging Target - Scintillator Screen

The Modulation Transfer Function (MTF) is a more standard method of resolution measurements and provides a more objective measure of the screen resolving capability. The MTF is produced by imaging an abrupt transition such as an edge. The resulting edge response is called the line spread function. This function is fit to a smooth curve to eliminate noise from the data. After Fourier transformation, noise in the data can prevent the MTF plot from converging in frequency space. The derivative of the fit curve produces the point spread function which can be Fourier transformed to give the MTF. The MTF is then normalized and calibrated to the spatial frequency used in the testing and is generally plotted as a percent modulation as a function of the spatial frequency.

Producing an accurate edge for X-ray imaging proved difficult. A number of techniques were empolyed to obtain high contrast images of an edge that is sharper than the screen and imaging optics resolving capabilities. The edge itself and its alignment relative to the $\mathrm{X}$-ray beem need to be optimized to produce an accurate MTF measutement. A microfocus X-ray source was used for low energy testing. The X-ray head exhibited a beam focus size of 10-50 micrometers depending on the voltage used. This small beam is in effect a point source, and does not degrade the edge image as seen by the detector.

Several approaches were used to obtain the line spread function. Initially, a knife edge was used to approximate the transition much the same way as in conventional optical testing. It was quickly determined that the X-ray beam penetrated far too much of the edge for the technique to be of value. A much more dense tantalum blade was made and later polished to produce a sharp edge. Alignment of the edge is also important, since a small tilt produces a thin section that X-rays can penetrate and blur the edge image.

One approach used to align the edge was to attach a small pinhole (25 micrometers) that was aligned by observation with a high-power microscope. The assembly was then mounted with a precision tip/tilt stage and aligned with the X-ray beam for a minimum edge transition region. The pinhole can just be discerned in Figure 7, which also shows the round pinhole holder and the top of the tantalum edge.

The tantalum edge improved the results in measuring the MTF of the screens. However, to further explore high-contrast imaging, a gauge block was aligned on edge and imaged. Gauge blocks have very well defined edges that are polished and flat to very high 
precision. Figure 8 shows the method of edge alignment. Two small plates with precision notches (250 micrometers diameter) were attached to the gauge block using a microscope. One plate was located on the front and the other on the rear of the gauge block. When placed in the tip/tilt mount, proper alignment could be obtained when both notches were at precisely the same vertical location.

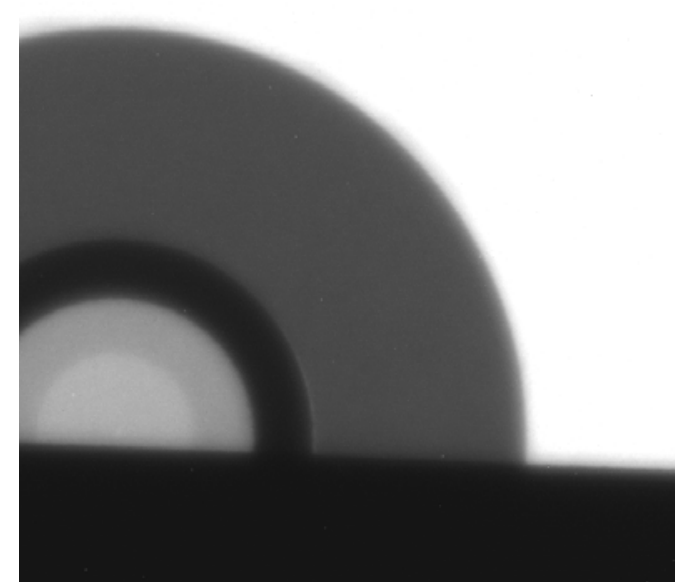

Figure 7. Pinhole edge alignment

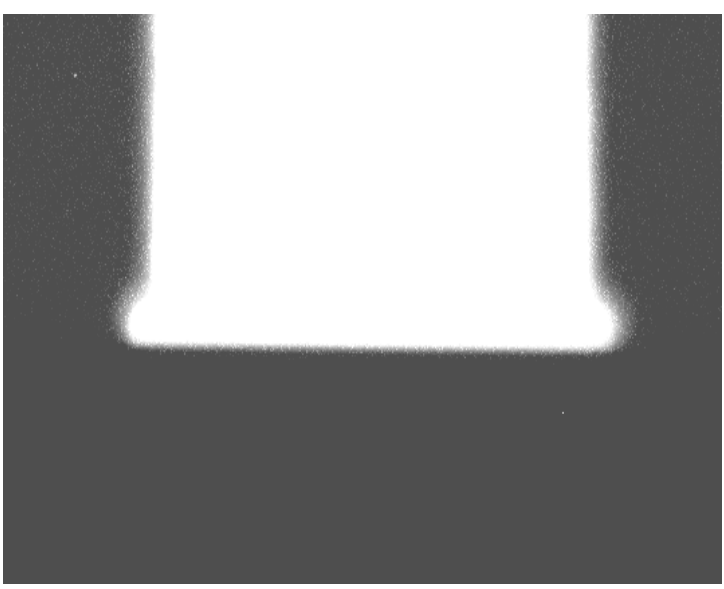

Figure 8. Front/back notch edge alignment

Results of the various methods of producing the line spread function are shown in Figure 9. The highest response obtained can be generally taken to be the most correct. A precision chrome-on-glass edge was imaged under white light conditions to produce the optical response function in absence of the X-ray screen. The chrome on glass curve shows that the system could image targets in excess of $20 \mathrm{lp} / \mathrm{mm}$ and not limit the response of the scintillator screens.

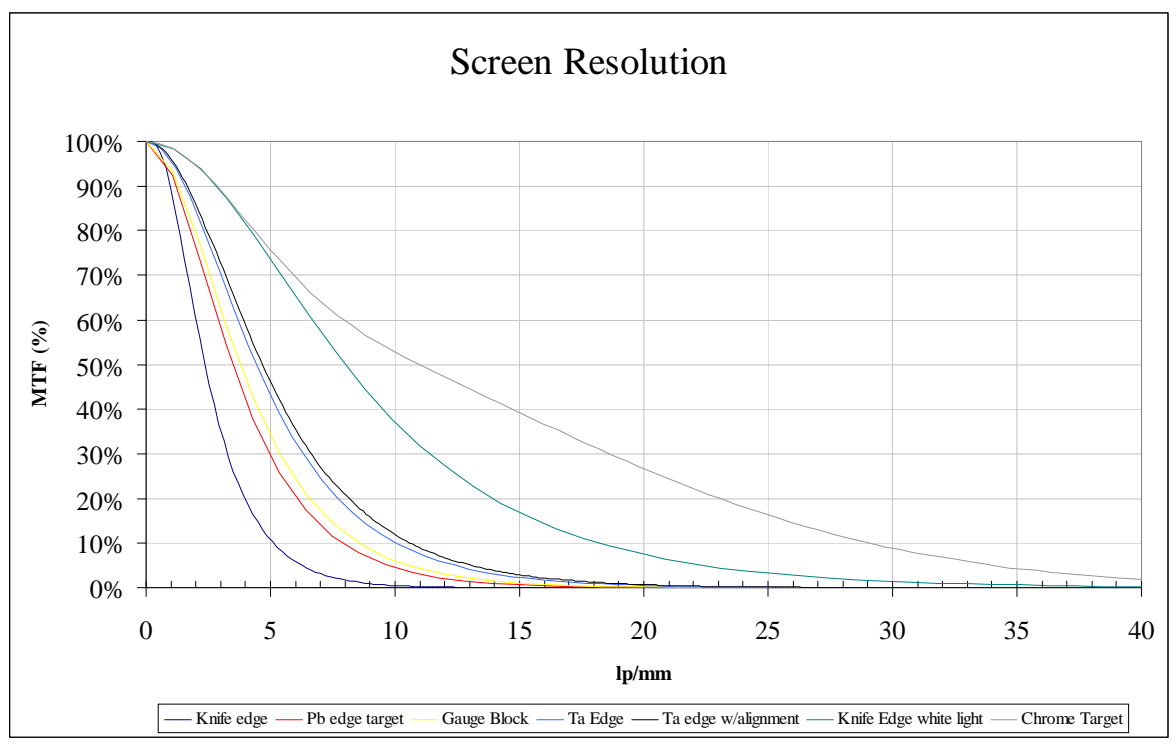

Figure 9. MTF calculations using a variety of line spread function targets 
A number of MTF tests were performed to determine the resolving power of the various screen materials at different energies. Figure 10 shows the MTF for several screen materials evaluated at $50 \mathrm{KeV}$. Although the CsI screen proved much brighter than the other candidates in earlier testing, it can be seen that it does not produce the best resolution.

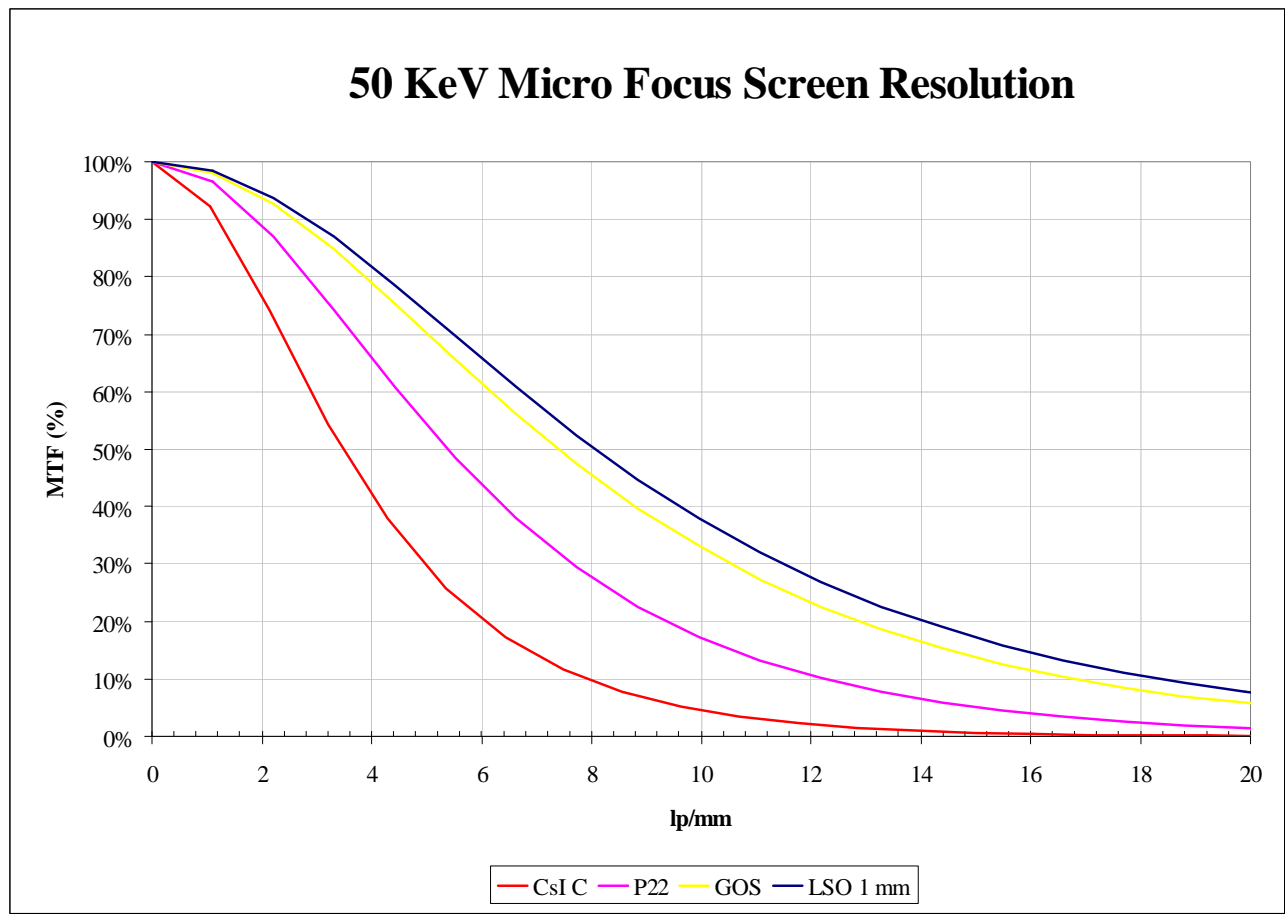

Figure 10. MTF calculations using for materials at $50 \mathrm{KeV}$

Several samples were so dim that resolution testing was not possible at $50 \mathrm{KeV} \mathrm{X-ray}$ energy. Using a Phillips $420 \mathrm{KeV}$ Bremsstrahlung source, a series of measurements were made to determine the spatial resolving capabilities of certain screen materials. Figure 11 shows the IQI, LSO and CsI-Al screen samples measured at $120 \mathrm{KeV}$ source peak voltage. The IQI and LSO samples were quite similar and both proved to exhibit higher resolution than the CsI screen. Thickness of the IQI and LSO samples were $6 \mathrm{~mm}, 1 \mathrm{~mm}$ respectively, and the CsI sample was a film deposition. 


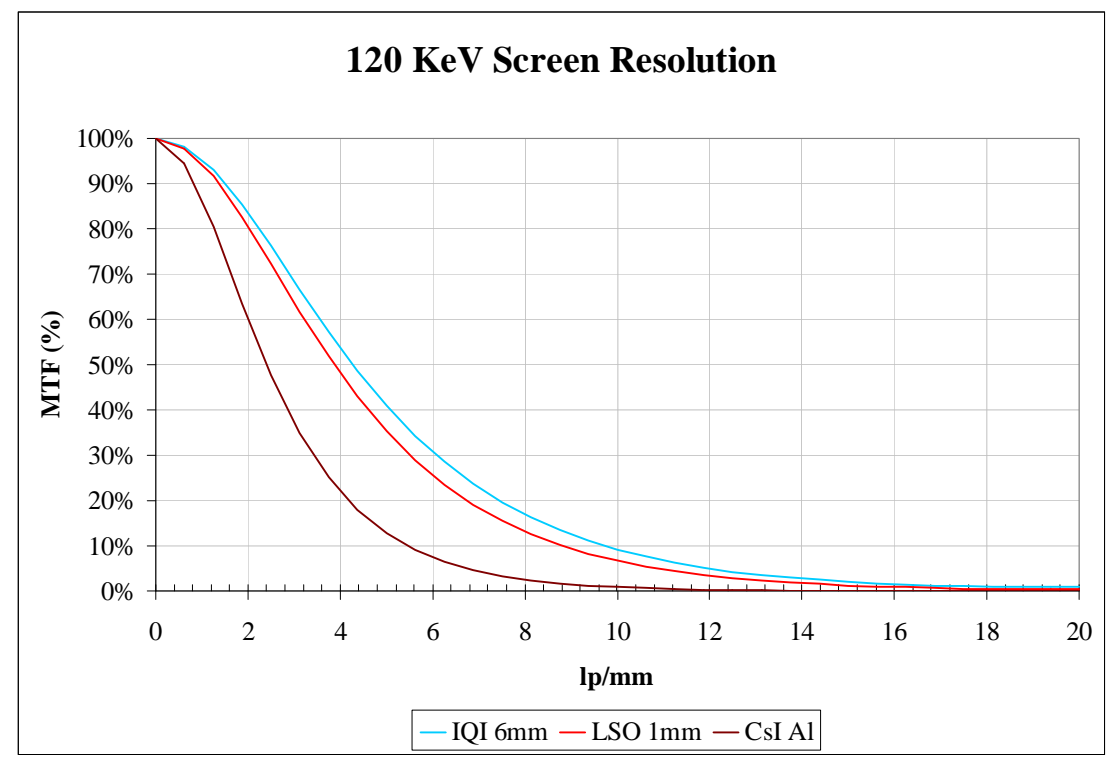

Figure 11. MTF calculations using for materials at $120 \mathrm{KeV}$

The same measurements were made at $320 \mathrm{KeV}$ and $420 \mathrm{KeV}$ to rank the various screen materials at higher voltages. The IQI screen does not degrade significantly from 120 to 320 as seen in Figure 12. However, the LSO and CsI screens have lost some resolution. The same trend is also seen as the voltage is increased to 420 in Figure 13, and a general degrading of the resolving capabilities of the screens begins to be apparent.

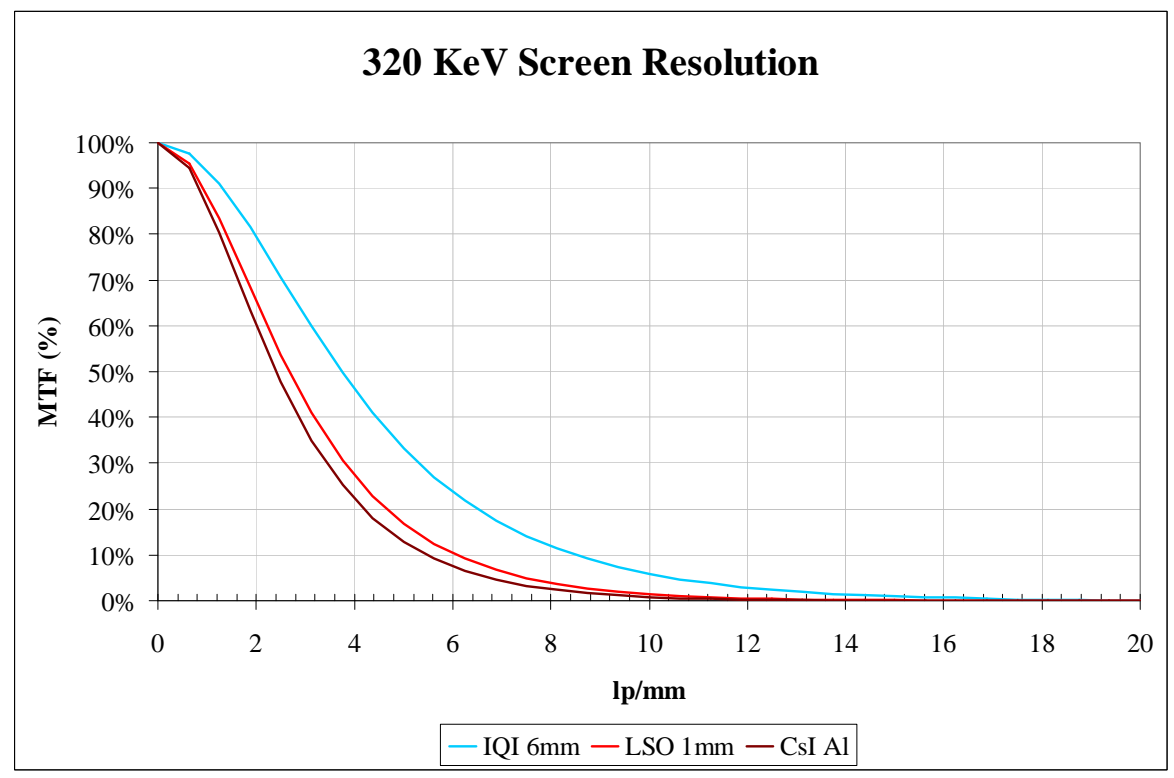

Figure 12. MTF calculations using for materials at $320 \mathrm{KeV}$ 


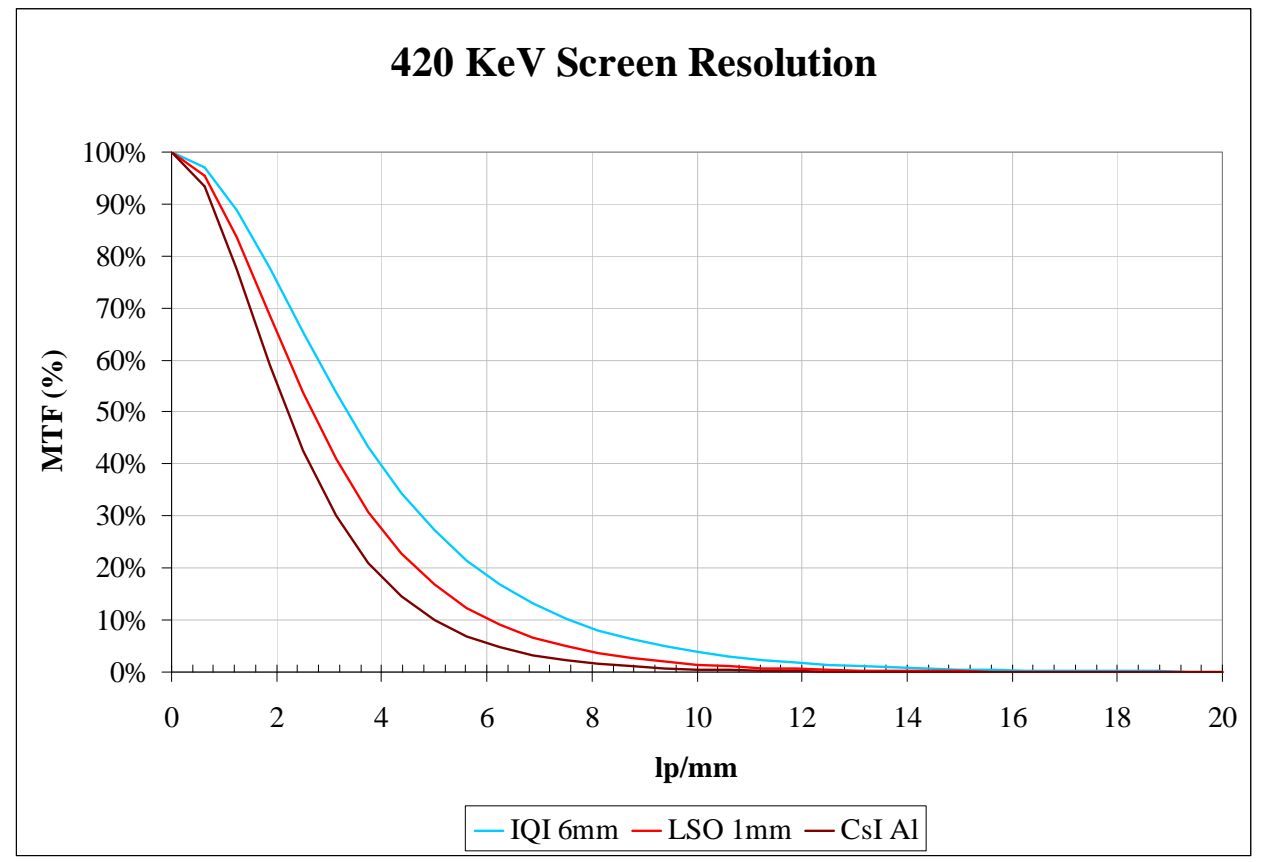

Figure 13. MTF calculations using for materials at $420 \mathrm{KeV}$

Figures 14-16 show the various screen materials as a function of the X-ray peak voltage. Interestingly, some materials such as the IQI and LSO show much more degrading of the resolution at higher voltages than the CsI.

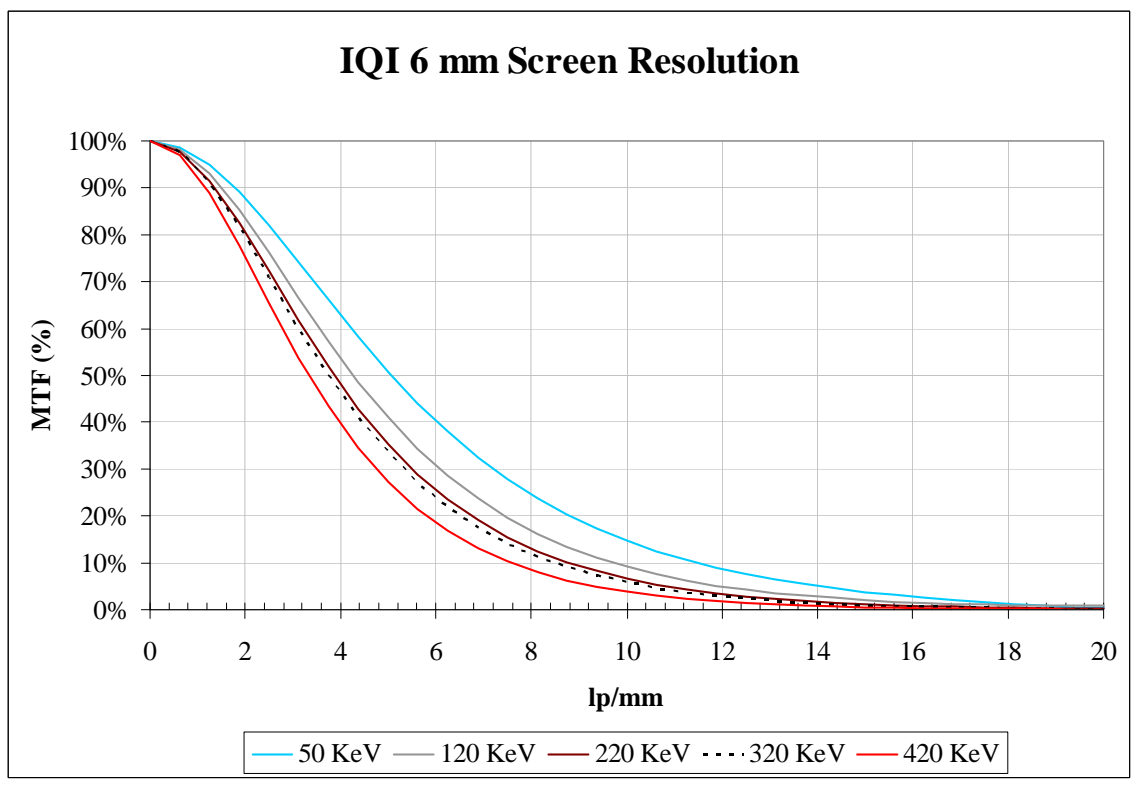

Figure 14. MTF calculations for the IQI screen 


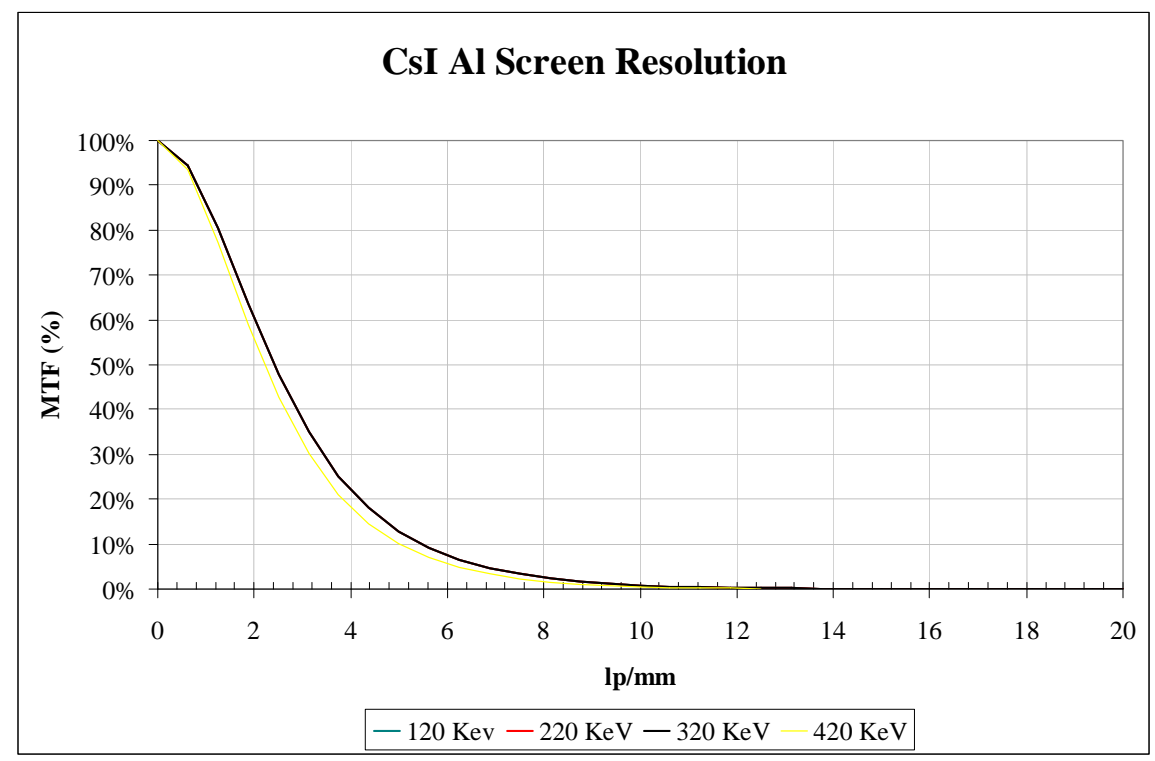

Figure 15. MTF calculations for CsI screen with Al filter

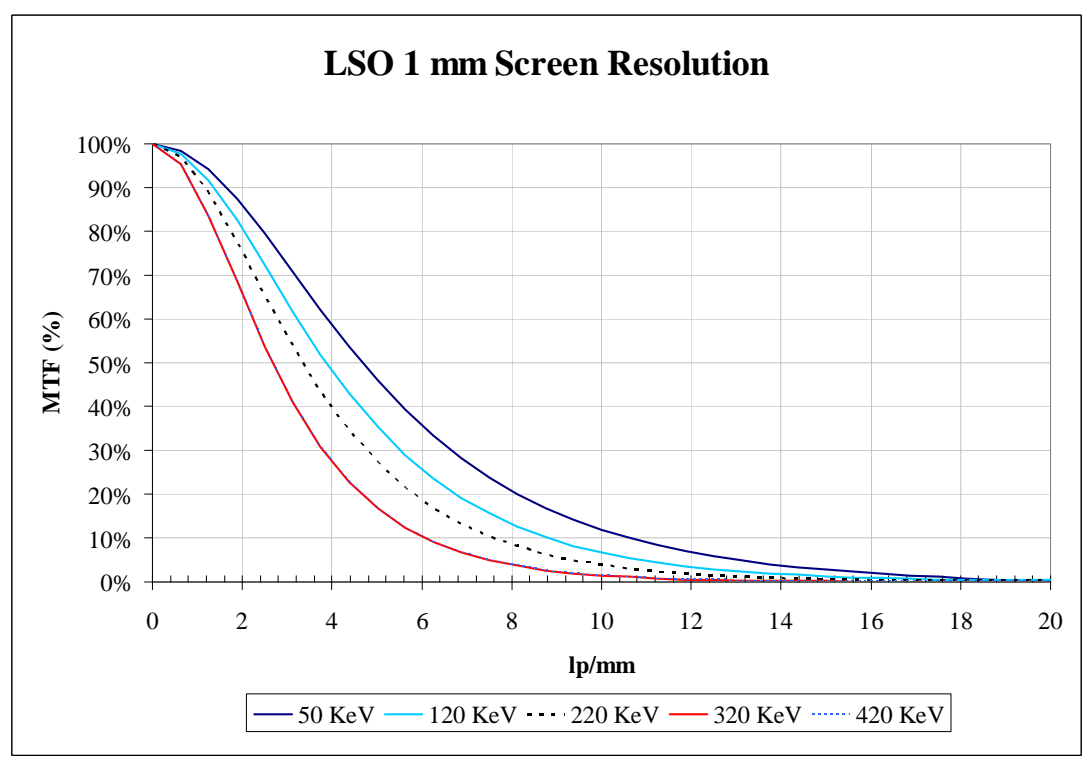

Figure 16. MTF calculations for the LSO screen

\section{CONCLUSIONS}

A number of significant findings were obtained from this study. Most important of the findings was that materials are commercially available that are much brighter than screens currently in use. This finding meets the original objective of the project.

Two objectives of the study; however, were not met. We hoped to evaluate a "quantum dot" (nanometer-sized particles of semiconductor material) wavelength conversion screen, but the manufacturer ceased production of the screen shortly before the project 
was started. The dot screen could be efficient in converting ultraviolet light to visible light which would have proved important for utilizing a Cherenkov screen. Since this was a very new, cutting-edge technology, an alternative supplier was not found during the study. Also, high-energy testing of a Cherenkov light screen was not performed due to difficulties in obtaining appropriate approvals for locating test equipment in the highenergy X-ray vault at Y-12. The test is still important, and is being pursued through follow-on funding sources.

Although many film shots will be eliminated by the availability of high quality digital images, the largest potential gains result from the availability of clearer images that show fine detail in the parts under analysis. Digital radiographic data also offers the possibility of easily sharing data with other sites. This could prove invaluable when critical material, placement, assembly, or quality issues are pressing. Also, increased throughput in the NDE facility allows statistically significant numbers of units to be analyzed. Digital technologies may in fact be needed just to meet minimum requirements of future demands. Increased brightness screens allow for such innovations as 3-D tomographic images to be acquired in a reasonable time. Much of the skill required to interpret "flattened" X-ray images is not needed to maneuver around the reconstructed tomogram.

This study showed that several commercially available materials are much brighter than screens currently in use. The study also showed that materials other than the brightest may be preferred for the best resolution. Since the X-ray conversion screen can be designed for easy exchange, a combination of materials will ultimately be the best approach for the broad range of radiography analysis performed at Y-12.

\section{ACKNOWLEDGEMENTS}

The authors acknowledge the advice and support of J.R. Ferguson in the Y-12 Product Testing organization for supplying high-resolution CCD's to make the resolution measurements for this study. Also, we acknowledge R.S. Steele and the Y-12 Plant Directed Research, Development, and Demonstration program for project support. 